2

3

4

\title{
Efflux Transporter
}

Shirin Jamshidi ${ }^{\dagger}$, J. Mark Sutton+, Sara Jambarsang ${ }^{\ddagger}$ and Khondaker Miraz Rahman ${ }^{+*}$

${ }^{\dagger}$ Institute of Pharmaceutical Science, School of Cancer and Pharmaceutical Sciences, King's College London, Franklin-Wilkins Building, 150 Stamford Street, London, SE1 9NH, UK +Public Health England, National Infection Service, Porton Down, Salisbury, Wiltshire, SP4 0JG, UK

${ }^{\ddagger}$ Department of Biostatistics and Epidemiology, School of Public Health, Sadoughi University of Medical Sciences, Yazd, IR

*Corresponding author: k.miraz.rahman@kcl.ac.uk

\section{Abstract}

In this study, we evaluate the ligand-induced structural rearrangements and conformational changes of $M e x B$, in relation to the protonation state of critical acidic residues, using tetracycline (TET) as a model substrate and phenylalanine-arginine beta-naphthylamide $(P A B N)$ as a model inhibitor.. We find that ligand binding leads to significant differences in the functional motions of transmembrane helices in conjunction with the multi-binding site. Ligand binding and subsequent extrusion or inhibition events are the consequence of various conformational changes in pump structure. Substrate binding affects conformational changes in a way that promotes the switching mechanism and efflux process. In contrast, inhibitor binding disturbs the switching mechanism and inhibits the pump by prompting different conformational changes in the protein structure. This study suggests that the Asp566 residue plays a critical role in the rearrangement of the transmembrane domains in the ligand-bound state.

\section{Keywords}

MexB efflux pump transporter, molecular dynamics simulations, conformational changes, Ligand binding, phenylalanyl-arginine-beta-naphthylamide, tetracycline. 
Pseudomonas aeruginosa is a major opportunistic pathogen and a leading cause of hospitalacquired infections and mortality worldwide ${ }^{1}$. It displays notable degrees of intrinsic resistance to a wide variety of antimicrobial agents, including most $\beta$-lactams, tetracyclines, chloramphenicol and fluoroquinolones. Efflux pumps present in $P$. aeruginosa play a significant role in conferring this resistance upon the bacterium ${ }^{2-7}$. Overexpression of RND (Resistance-Nodulation-Division) superfamily efflux pumps in Gram-negative bacteria is a primary component of multidrug resistance (MDR) ${ }^{8}$. This type of efflux pumps recognizes a diverse range of compounds and harness the proton-motive force (PMF) to pump them out of the bacterial cell. MexAB-OprM, an RND-type efflux pump, is one of the major efflux pumps that operate in $P$. aeruginosa and confer resistance to antimicrobials ${ }^{9}$. It is a tripartite pump consisting of an inner membrane spanning transporter MexB, a trimeric lipoprotein OprM that associates with outer membrane and a hexameric membrane fusion protein MexA that forms a stable complex with MexB at the inner membrane ${ }^{6}$. The MexB subunit is a large protein with a molecular mass of $113 \mathrm{kDa}$ and has 1,046 amino acid residues. It consists of a transmembrane domain, a porter domain and a funnel-like (FL) domain. The transmembrane domain has $12 \alpha$-helices and the porter domain contains the drug-binding site while the FL domain interacts with other components of the transporter ${ }^{10-12}$. The large loop like structures present in MexB interacts with both MexA and OprM to form the functional pump assembly ${ }^{13-}$

17. The transmembrane domain contains five charged amino acid residues and three out of these five residues are highly conserved in RND superfamily of efflux pumps. The specific location of these highly conserved charged residues in the transmembrane domain suggests important role of these residues in proton conduction ${ }^{11}$. 
The substrate specificity and selectivity of the MexAB-TolC efflux pump depends on the homotrimeric structure of MexB. It has a threefold asymmetric conformation in which each monomer adopts a different conformation (access, binding or extrusion) ${ }^{18-24}$ and employs a allosterically coupled rotation mechanism wherein each monomer successively adopts one of the three aforementioned conformations ${ }^{20,21,24-30}$. The proximal and distal binding pockets that are involved in forming the multi-binding site of MexB play critical roles in determining its substrate specificity and binding ${ }^{31-40}$. The distal binding pocket includes many hydrophobic, polar and charged residues, and this microenvironment mediates the extrusion of a wide range of compounds by RND superfamily pumps ${ }^{41-43}$.

Using X-ray crystallography and computational simulations, Eicher and coworkers ${ }^{44}$ showed a collective motion between conformational changes of the transmembrane domain for protons and pore domain at the periplasmic area for ligands in three protomers of main components of RND efflux pump, AcrB ${ }^{44}$. The mechanism of proton-coupled drug-efflux in RND superfamily transporters has been described as the collective motion. In this study, by considering the same protonation states of the specific residues of the transmembrane domain detailed in the aforementioned study, we show the effect of ligand binding on the conformational changes with the help of principal component analysis (PCA) and by monitoring the density of distances of transmembrane helices in the protomers. We identify several essential motions and functional dynamics and describe the conformational changes that govern distinct access, binding and release states to gain insight into the efflux or inhibition mechanism in the presence of a ligand. The results of this study that connects structural and functional aspects could set the stage for inhibitor and new type of hybrid efflux resistant antibiotic design strategies that can explicitly target mechanistic features of a particular pump. 
Results

Important regions of MexB. Table S1 details the residues in MexB that interact with the ligand during MD simulation trajectories. They have been marked by sequence alignment of MexB from $P$. aeruginosa to the solved structure of AcrB from Escherichia coli ${ }^{19,45}$. The matched amino acids in the MexB and AcrB sequences occupy the same locations in their corresponding PDB structures. Additionally, the protonated residues in the transmembrane domains ${ }^{44}$ of the different monomers of the protein are listed.

Principal component analysis. Principal component analysis (PCA) was performed as a postMD analysis to characterize the conformational transitions in the MexB transporter, modulated by the inhibitor (PABN) and substrate (TET) (Fig. S1). As Fig. 1 indicates, different patterns of conformational changes in the systems revealed different dynamics and functions between the free form of the MexB transporter and the transporter in complex with either the inhibitor or the substrate. Ligand binding clearly affects the conformational changes of the transporter, with different ligands prompting distinct conformational changes.

Correlation network analysis. The motions of the residues of the diverse protomers were monitored using the first mode of PCA to understand the essential dynamics for uncovering functional differences and their mechanisms. For the sake of simplicity, they are shown in two graphs which have been categorized differently (Fig. S2 and S3). PCA on MD data was useful for finding the protein segments that were most involved in structural changes. The RMSF (root mean square fluctuations) calculation was used to determine how much each residue moved during the trajectory and how much each residue contributed to a principal component. This enabled us to define a correlation in the motion of the essential parts of the efflux pump after complexing with the ligands was observed. Comparing with other systems, 
the fluctuations in the binding monomer of the PA $B N$-bound complex were slightly restricted, especially in critical regions like the tip residue Phe617. However, diverse parts of the protein show a distinct correlation to the tetracycline-bound and ligand-free states of MexB.

From the plots in Fig. S2, it can be observed that the access, binding and release protomers are the protomers with the most peak fluctuations in the ligand-free MexB, MexB-PA $\beta N$ and MexB-TET systems, respectively. The highest and lowest functional motions were seen in the access and release protomers, respectively, whilst the binding monomers show the highest degree of correlation among diverse parts of the protein between the different efflux pump systems. Also, considering the peaks of the fluctuations in each of the graphs of the binding protomer, the highest degree of correlation among the different regions can be observed in the MexB-PABN complex, with most peaks in diverse regions of protein, and the lowest correlation belongs to ligand-free MexB, with few peaks on the corresponding graphs (Fig. S2). However, the various graphs clearly show different dynamics in the different systems and even within protomers of an individual system. These graphs indicate that the G-loop (Phe617) is much more flexible in the binding and release monomers of ligand-free MexB compared to MexB-PA $\beta N$. The same region also shows considerable fluctuations in the case of MexB-TET (Fig. S3).

Distance density distributions: Substrate and inhibitor binding induce conformations of MexB which are distinct from the free form of the protein., Monitoring the distances between protonating residues in transmembrane helixes, during MD simulations, was transformed into distance density distributions which characterize the spatial relationships between pairs of transmembrane helices in the diverse protomers of MexB (Fig. 2). The distances between the protonated residues in each helix of the transmembrane domain (marked below) were 
considered to monitor the relative orientation of the helices during conformational changes in different systems.

Also, by considering Fig. S2 \& S3, the protomers with highest RMSF in several helices of the transmembrane (TM) domains are represented in Table S2. As shown by the data, the access, release and binding protomers are the protomers with the highest fluctuation and functional dynamics in the ligand-free, PABN-bound, and TET-bound forms of MexB, respectively.

The results show that the distance density peaks between different TMs are clustered in ligand-bound states, compared with the free form of MexB in which the distances showed a broad range of distribution (Fig. 3). Broad distributions in TM10-11 are observed among diverse protomers with average distances of 18,58 and $56 \AA$ for access, binding and release distances of 27, 26 and $29 \AA$ in binding protomers of MexB, MexB-PABN and MexB-TET, those of the ligand-bound structures reveals considerable differences in structural changes upon the binding of different ligands. These are primarily observed at i) TM4 and TM10 (Fig. 
helices of the transmembrane domains, at ii) TM2 and TM11 (Fig. 3, row 2), which border the bilayer, and finally iii) the turn connecting TM7 (TM7t) and TM10 (Fig. 3, row 7). TM7t includes key residues involved in transferring functional dynamics between the transmembrane domains and pore domains that includes the multi-binding site. Importantly, diverse pairs of TMs helices represented in Fig. 3, include the protonated residues that are essential to the proton translocation function of the transporter as a proton motive force nanomachine. In contrast, minor rearrangements along the interface of TM4 and TM11 are observed in the density distance distributions (Fig. 3, row 3). Significant changes in the distances with the largest density and the width of the distance distributions are observed along with the turn connecting TM7 (includes key residues of Asp566). Most substantial changes occurred at TM10-TM11 (Fig. 3, row 8) with restricted mobility observed in almost all the monitored helixhelix interfaces. Therefore, we consider that these distance changes reflect functional dynamics of diverse conformations, that proceed by a rearrangement of the TM helices that starts from the turn connecting TM7t end to TM11. The whole path of dynamics proceeds by ligand binding that affects the distal binding site conformational changes, by rearrangement of the location of G-loop and then Ser loop that finally transfers to TM7t. This path can happen, conversely, from the distal site by ligand binding to the transmembrane domain via the turn, including Asp566.

Density maps of distances, represented in Fig. 3, confirm the extensive ligand-dependent rearrangements in the complex structures. In particular, the lowest monitored distance around 8 Å between amino acid Asp566 of TM7t from Asp923 of TM10 (Fig. 3, row 7) suggests an essential role for binding monomers in the conformational changes toward the function of the efflux pump. Notably, ligand binding restrains the distance distributions (Fig. 3). Also as represented in Fig. S4, the average distances involving Asp566 show more variety than the 
monitored averages for other amino acids. This indicates the effect of ligand binding on the location and orientation of Asp566 to Asp923 and, consequently, the rearrangement of the turn connecting TM7 and TM10 with respect to each other. Table 1 clearly shows the density of distance between TM7t and the binding site is slightly farther $(22.04,22.55$ and $22.78 \AA$ in ligand-free, $\mathrm{PA} \beta \mathrm{N}$-bound and TET-bound MexB, respectively) whereas the distance between TM7t and TM10 is slightly closer in the binding monomer of ligand-bound systems (8.74 and $8.55 \AA$ in PAßN-bound and TET-bound MexB, respectively) compared with the ligand-free system ( $8.75 \AA$ ). It can lead to rearrangements along the rest of the TM helices. The different natures of the rearrangements along the various TM helices of the systems reflects the effect of ligand binding and the type of ligand. Consistent with this conclusion, the density of distance distributions when the system is ligand-free are broad, suggesting greater flexibility of the backbone. Substantial amplitude distance changes are observed in TM2-TM10 and TM2-TM11 (Fig. 3, rows 1 and 2), indicating extensive ligand-dependent repacking. Although the exact nature of the underlying structural rearrangements is challenging to infer from the data, distance densities suggest a flow of conformational rearrangement among the TM helices with a central role of Lys939 (TM10), Thr976 (TM11) and Asp407 and Asp408 (TM4) in conferring conformational changes, promoted by ligand binding in the multi-binding site of the transporter. This agrees with previous observations by Takatsuka and Nikaido that Thr978, Lys940, Asp407 and Asp408 in AcrB are essential to adopt conformational changes towards the functional dynamic of the transporter ${ }^{46}$. Also, according to the current study, Asp407 or Asp408 of TM4 and Lys939 of TM10 show short distance components to Thr976 of TM11 in the ligand-free and ligand-bound states (Fig. 3 and Table 1). Conversely, the lower density and/or broader range of distance in the ligand-free distributions (Fig. 3) suggest a highly flexible conformation with extensive fluctuations, that is in a good agreement with 
RMSF graphs (Fig. S2 and S3). Specifically, more fluctuations are seen within the TM helices and multi binding site regions in ligand-free form compared with ligand-bound systems.

The penetration of Asp566 toward TM10, implied by the shorter distance compared with the ligand-free state, presumably confers the conformational changes in transmembrane domains in different ways in PAßN-bound and TET-bound systems. The domain including the G-loop is connected to Asp566 through a super secondary structure of beta-alpha-beta motifs that has a crucial role in forming the hydrophobic core of the pump. Binding of ligands to the protein causes Asp566 to undergo a closing motion upon ligand binding, evidenced by the shift in the density of distance (Fig. S5). In the next stage, ligand binding affects transmembrane conformational changes by the proximity of Asp566 to Asp923 which are protonating residues in $\mathrm{TM} 7 \mathrm{t}$ and $\mathrm{TM} 10$ respectively.

However, the density distributions at sites containing of Asp566 and Asp923 show the presence of a trend of focusing and reducing distance. This movement is attenuated near the end of the TM10 helix. Except for the distance between Asp566 and Asp923 in TM7t-TM10 of the binding protomer, most of the distances in the transmembrane domains were characterized by broad distributions, indicating a highly dynamic backbone (Figs. S2 and S3). The conformation is stabilized by ligand binding and is slightly reduced in MexB-PABN with greater correlation between different regions of the transporter. Phe617 is in direct contact with the bound ligand in the complex structures during MD trajectories. Moreover, the Gloop forms part of a hydrophobic groove, on one side, and it is involved in inhibition by affecting Asp566 location through the beta-alpha-beta motif connection on the other side. Asp566, can be identified as a critical residue linking the ligand-binding conformational changes to the transmembrane domains, and the other key residues in the multi-binding site by monitoring the distance density between Asp566, and the other key residues in the multi- 
binding site (Fig. 4). Notably, Asp566 shows a broader range of distance density to the multi-

binding site residues in the access protomer compared to the binding and release protomers. Also, it was observed that the graphs of ligand-bound states in diverse monomers showed more variety, when compared to each other, than those in a ligand-free state. This likely reflects the direct effect of ligand binding on conformational dynamics.

Monitoring the distance between the ligand and the key residues of the passageway in the multi-binding site in different complex systems (Fig. 5) shows that the range of density distributions are broader in the MexB-PA $\beta N$ complex compared with the MexB-TET complex.

The density of distances is restricted to a certain value in the MexB-TET complex, which is larger than the corresponding values in the case of MexB-PA $B N$ complex. It clearly shows that $\mathrm{PA} \beta \mathrm{N}$, as mentioned above, could occlude the passageway and could affect the conformational changes of MexB in a way that means the conformer switching mechanism is distorted more than for tetracycline binding. This result is in accordance with the PCA analysis (Fig. 1) in which the graphs of conformational changes in the TET-bound complex is more similar to the ligand-free system than the PABN-bound one.

Also, monitoring the distance between the residues forming the proposed gate and cleft (Fig.

6) showed that ligand binding significantly affects the distance density in ligand-bound states compared with the ligand-free form. The access, binding and release monomers confer diverse density with different pattern in the distance between cleft and postulated gate residues. According to the graph, PABN binding gives a broader range of change with almost same values in the distance range of ligand free form but still noticeable difference in density 
distribution in binding and access protomers whereas TET binding decreases the range in

binding and shifts the range toward higher values in release protomer considerably.

To investigate the MexB structure under ligand binding conditions that are expected to promote diverse conformations, we compared the highest points of distance density distributions, represented in Table 1, to the corresponding distances in the crystal structure of MexB (Protein Data Bank identity code 3W9) and MD simulated ligand-free form separately

(Fig. S6). The crystal structure was considered to provide a general reference for the conformation, so the right panels were assigned as the reference to monitor and compare the discrepancy of the distance density with certain distances in the crystal structure. As the left panel shows, the graphs for release protomers are almost superimposable and aligned with each other. However, there is a considerable difference between two complexes when the binding and access protomers are considered. The discrepancy remains after subtracting the references, clearly showing that ligand binding could lead to a specific dynamic function that results in efflux in the case of the substrate and inhibition in the case of the inhibitor. It seems that differences in protonation states of specific residues in the access and release protomers could efficiently drive the conformational changes in the monomers by adjusting the distances between the TM helices and affecting their orientations to each other. The differences in conformational changes in binding and access protomers, despite the similar protonation state in certain residues, confirms the key role of ligand binding on TM helices repacking and its role in consequent conformational changes. Protonation invariably leads to discrepancy among TM helices in the diverse protomers of the different systems. Therefore, different rearrangement in the transmembrane domains of the transporter occur because of the ligand binding. 
Distance density distributions of helices TM2, TM10, TM4 and TM11 in the binding protomers suggest that TM10 and TM11 undergo rearrangement of the transmembrane domain when a ligand is bound. The TM4 and TM10 rearrangements are promoted by ligand binding and likely facilitated by the protonation state in Asp407 and Asp408. These residues tend to take a proton from Lys939 at TM11 which leads to changes in the conformational dynamics of the corresponding TM helices in binding and access monomers. The conformational changes at the binding site extend to the loop connecting TM7, linking it to the TM helixes.

The right panels of Fig. S6 in the linear view (Fig. S7) indicate a significant shortening in the intra-residue differences in the binding protomer of MexB-PAßN (e.g. 1, 2, 3, 4, 14, 19, 20, 25, 27, 29 and 47) that imply a large-scale movement of the helices. This is an indicator for occlusion of the transmembrane domain and binding site in the presence of the inhibitor. Although prominent ligand-induced changes in the distance distributions are observed in the transmembrane domains of TM2, TM10, TM4 and TM11, they are generally smaller in magnitude and no discernible pattern was evident from the comparison of changes in other helices. Given that these TMs are involved in conformational changes and consequently function, it is not surprising that the rearrangements of TM helices with specific protonated residues, necessitate rearrangement at the transmembrane domains.

Overall, the density distributions reveal several trends consistent with three distinct conformations corresponding to the inhibitor-bound, substrate-bound, and ligand-free MexB complexes. First, we can observe changes in the distance density as well as the width of the density distributions between the diverse conditions which unequivocally demonstrate extensive conformational rearrangements. Second, the shape of these distributions implies that the substrate-bound and inhibitor-bound states are ordered in stark contrast with each 
291 other and with the highly dynamic ligand-free state, with a broader range in the distribution 292 values. The ligand-free form of MexB shows a wide range of density distances, that it is much 293 more restrained, or which shows a higher density in ligand-bound states of MexB. Also, the 294 effect of ligand binding is considerable in the distances between TM helices of MexB in the 295 different systems after extracting them from long MD simulations trajectories.

296 
To provide a global perspective on the conformational changes and structural rearrangements of MexB, the changes in the distance density were plotted as a function of the distances. It has been narrowed down to monitor just the critical residues belonging to the essential regions of the protein that were expected to be involved in functional dynamics. Nevertheless, this exercise provides the qualitative visualization of the regions of conformational change, thereby identifying a complex web of structural rearrangements focused on TM2, TM4, TM10 and TM11 and the turn connecting to TM7. The ligand-free form of MexB has been considered as a reference to interpret the diverse analysis in a structural context. The data showed extensive differences between the ligand-free and the ligandbound complex conformations in MexB.

In this study, we have gained insight into the differential function of MexB in the presence of substrates and inhibitors. The results show that ligands induce motion in the transporter through their interactions with the multi-binding site, but that this motion is liganddependent and thus different for substrates and inhibitors. To this end, the conformer patterns adopted by the TET-bound form are not observed in the simulations of the PABNbound transporter. This type of motion dependency has implications for understanding the effects of conformational changes on ligand binding by MexB and in defining how inhibitors mediate their effects.

Ligand binding affects the rotation and tilting of TM4-TM10, TM2, and TM11, which together modulate either the efflux or the inhibition pathway. Asp566 which is in the TM7 connecting loop between the binding site and transmembrane helices, emerges as a central amino acid 
residue in this conformation switch, undergoing extensive rearrangement in diverse protomers. The importance of Asp566 to the conformational switch was confirmed, acting primarily through extensive rearrangement of TM2, TM4, TM10 and TM11.

The flexibility of Asp566, presumably a consequence of the ligand binding, enables large-scale reconfiguration upon concurrent ligand binding and protonation of Asp566. The rearrangements of the backbone and side chains of TM helices may lead to a different conformational change, consequently resulting in a different function. Ligand binding induce the rearrangement of the TM helices, which participate in the functional mechanism. TM helices undergo significant amplitude movement coupled to extensive rearrangement of the transmembrane domain. This movement is controlled both by the diverse protonation state of Asp407, Asp508, Asp566 and His338 in diverse protomers and the type of ligand bound simultaneously.

In brief, this study showed that ligand binding extensively affects the conformational changes leading to a specific dynamic of the MexB transporter that results in either efflux or pump inhibition. The results of this study include detailed data on the atomic level that could help elucidate the mechanism of action of tripartite efflux pumps and consequently inform the development of better inhibitors of RND efflux pumps by rational drug design, in turn helping to overcome antibiotic resistance in multidrug-resistant Gram-negative pathogens. 


\section{Methods}

MD simulations were performed using the program AMBER 16 package program ${ }^{47}$. MD simulations started from the docked structures, obtained from blind docking by AutoDock SMINA, which followed by flexible docking using the GOLD ${ }^{48}$ program. In this study, the power of GPU acceleration was used to study conformational transitions that occur in MexB in free form and interaction with substrate and inhibitor, separately. Also, the CUDA implementation of PMEMD was used to carry out the simulations on GPUs.

System setup. The crystallographic structure of Pseudomonas aeruginosa MexB transporter was obtained from Protein Data Bank (PDB ID code 3 W9I with a resolution of $2.71 \AA^{49}$ in the Loose/Tight/Open (LTO) asymmetric conformation as the starting conformation for the study. The residues not resolved by $\mathrm{x}$-ray were added to amend the structure using Biovia Accelrys Discovery Studio. The most probable protonation state of specific residues in the access (Loose/L) - binding (Tight/T) - release (Open/O) transition was changed. These include Asp407 and Asp408, in the core of the TM domain, which is ionized in the L and T states but becomes protonated in the $\mathrm{O}$ state. Interestingly, His338 at the periplasmic end of TM2 is protonated in the $\mathrm{O}$ state and becomes deprotonated in L and T states whereas Asp923 at the periplasmic end of TM10 is protonated in $\mathrm{L}$ and $\mathrm{T}$ states and becomes deprotonated in the $\mathrm{O}$ state; conversely, the neighbouring Asp566 in the region between TM7 and PC1 subdomain is most likely deprotonated in $\mathrm{L}$ and $\mathrm{T}$ and becomes protonated in the $\mathrm{O}$ state (Fig. S6). The previous calculations indicated that both Lys939, Arg971, and Asp976 remain protonated throughout the cycle. All these residues were adjusted to their protonation or deprotonated states in the corresponding protomer in the PDB crystal structure of MexB by following the literature ${ }^{44}$. The final structure was equilibrated and minimized using AMBER 16 package program. 

were generated by Chem3D 15.0 and were minimized using SYBYL software program. The force-field parameters for the ligands were developed following the Antech AMBER protocol of the AMBER package program.

Molecular docking. The starting structures for running simulations were obtained by molecular docking of the ligands to the MexB transporter. The orientation of the ligands within the multi-binding site of the binding protomer was taken from docking calculations performed with the AutoDock SMINA package through blind molecular docking, to find the particular cavity for ligands within the multi-binding site of the transporter with the best affinity among all the probable ones. All the parameters were kept at their default values for running SMINA. Then, an evaluated flexible molecular docking was performed using GOLD molecular docking into the SMINA-located binding site to find the best pose and orientation of the ligands in the located binding pocket. The Genetic algorithm (GA) is used in GOLD ligand docking to examine the ligand conformational flexibility, along with the partial flexibility of the protein. The maximum number of runs was set to 20 for each compound, with the default parameters (100 population size, 5 for the number of islands, 100,000 number of operations and 2 for the niche size). Default cut off values of $2.5 \AA$ ( $d H-X)$ for hydrogen bonds, and $4.0 \AA$ for van-der-Waals distance was used. When the solutions attained RMSD values within $1.5 \AA$, GA docking was terminated.

Molecular dynamics simulations. MD simulations of the free form and ligand-bound structures of MexB were carried out using the AMBER16 package program ${ }^{47,50}$. The topology and the initial coordinate files for these apo-protein structures were created using the LEaP 
module of AmberTools16 ToolKit program. The protein and docked complexes were successively embedded in 1-palmitoyl-2-oleoyl-sn-glycerol-3 phosphoethanolamine (POPE) bilayer patches, solvated with explicit TIP3P water model and neutralized with the required number of randomly placed $\mathrm{K}+$ ion. The ion count was suitably adjusted to account for an osmolarity of $0.15 \mathrm{M} \mathrm{KCl}$. Embedding of the protein systems into a pre-equilibrated POPE bilayer patch was performed using the PPM server and subsequently the CharmmGUI tool ${ }^{51}$. The lipid residue nomenclature was converted from the CHARMM to AMBER format using the charmmlipid2amber.py python script provided with AmberTools. The central pore lipids were then added after calculating the number of lipids to be added to each leaflet by dividing the approximate area of the central pore by the standard area per lipid of POPE molecules ${ }^{52}$. Periodic boundary conditions were used, and the calculated box dimensions from the water molecules were $162.17 \times 162.67 \times 197.35$.

For running molecular dynamics of lipid bilayer systems, including protein complexes, the 404 following protocol was used in an order; Minimization, heating 1 (holding the lipids fixed), heating 2 (holding the lipids fixed), 10X Hold to equilibrate periodic box dimensions, production with constant pressure. Energy minimization with a combination of steepest descent and conjugate gradient methods was carried out using the pmemd program implemented in AMBER16 to relax internal constrains of the systems by gradually releasing positional restraints. All the systems were minimized for 15000 cycles of steepest descent followed by 15000 cycles of conjugate gradient minimization. Following this, the systems were heated from 0 to $303 \mathrm{~K}$ by a 1 ns heating $(0-100 \mathrm{~K})$ under constant volume (NVT) followed by 5 ns of constant pressure heating (NPT) (100-303 K) with the phosphorous heads of lipids restrained along the z-axis to allow membrane merging and to bring the atmospheric pressure of the system to 1 bar. Langevin thermostat, with a collision frequency of 1 ps-1, 
was used to maintain a constant temperature, and multiple short equilibration steps of 500ps under anisotropic pressure scaling (Berendsen barostat) in NPT conditions were performed to equilibrate the box dimensions. A time step of $2 \mathrm{fs}$ was used during all these runs, while post-equilibrium MD simulations were carried out with a time step of $2 \mathrm{fs}$ under constant volume conditions after hydrogen mass repartitioning ${ }^{53}$. The particle-mesh Ewald (PME) algorithm was used to evaluate long-range electrostatic forces with a non-bonded cutoff of $10 \AA$. . During the MD simulations, the length of all R-H bonds were constrained with the SHAKE algorithm. The ff14SB ${ }^{54}$ version of the all-atom Amber force field was used to represent the protein systems, while lipid16 ${ }^{52}$ parameters were used for the POPE bilayer. After equilibration, $2 \mu$ s long MD simulations were performed for each system. Trajectory analysis was done using cpptraj module of AmberTools16, Bio3D, and VMD1.9.1, and density graphs were plotted using the R program.

Principal component analysis. To identify and highlight possible identities and differences in the collective motions of protomers, we determined the covariance matrices from the equilibrium trajectory and conducted a principal component analysis ${ }^{55,56}$. As conventional in principal component analysis, the covariance matrix was developed utilizing the threedimensional positional fluctuations of $\mathrm{C} \alpha$ atoms from their ensemble average position (after least-squares fitting to eliminate rotational and translational motion). Diagonalization of the covariance matrix produces a collection of eigenvectors and corresponding eigenvalues, which describe the direction and amplitude of the motion, respectively. The eigenvectors are then sorted according to the decreasing order of their associated eigenvalues, such that the first eigenvector describes the most substantial contribution to the total fluctuation of the system. To visualize the motions represented by the eigenvectors, the structures from the 
trajectories can be projected onto each eigenvector of interest (principal component or PC) and transformed back into Cartesian coordinates. The two extreme projections along each eigenvector can then be interpolated to create an animation or compared to understand which parts of the protein are moving according to that specific eigenvector and to what extent. Usually, a combination of the first few principal components can represent most of the collective motions, the "essential dynamics" 55 , occurring in an MD simulation among the different regions of a protein. This method has the benefit that the dynamics along the individual modes can be inspected and visualized separately, thereby allowing one to filter the main modes of collective motion from more local fluctuations.

Clustering of MD trajectories. For cluster analysis of the MD trajectories, the average-linkage hierarchical agglomerative clustering method implemented in cpptraj module of AMBER was used. Such clustering serves to decrease the number of structures for analysis, yet maintaining the large conformational space sampled during the MD runs. In this approach, for each system, the representative structures from each of the 10 top clusters produced were used to conduct quantitative analyses in order to account for dynamical behaviour.

Analysis of protein conformational change using principal component analysis (PCA). The original trajectory files produced by MD were significantly large, and we cannot include them in the Bio3D package installed in the $\mathrm{R}$ program due to space limitations, so the trajectories in each MD simulations were down-sampled with an interval of 50 . The points represented in the conformer plots were computationally clustered and coloured by the cluster. This was performed by creating a distance matrix of the principal components of interest. 
PCA reduces the dimensionality of large data sets by calculating a covariance matrix and its eigenvectors. Vectors with the highest eigenvalues become the most significant principal components. When principal components are plotted against each other, similar structures cluster, each cluster then theoretically represents a different protein conformational state. To avoid sample noise from random fluctuations ${ }^{57,58}$, following MD simulations of the MexB transporter systems, the PCA was calculated only for $\mathrm{C} \alpha$ atoms. Then, each protomer was selected in a separate PCA analysis, which was a good discriminator of conformations.

With the Bio3D package installed in $\mathrm{R}$, the plot command has been overloaded to create a default PCA plot with four graphs. Three are the z-scores of the first three principal components plotted against each other in two dimensions. The last is a scree plot representing how much of the variance of the data set is captured by each principal component.

\section{References}

1 Brown, M. R. W. Resistance of Pseudomonas aeruginosa. doi:http://doi.org/10.1002/jobm.19770170412 (1975).

2 Kohler, T. et al. Multidrug efflux in intrinsic resistance to trimethoprim and sulfamethoxazole in Pseudomonas aeruginosa. Antimicrob Agents Chemother 40, 2288-2290 (1996).

3 Li, X. Z., Nikaido, H. \& Poole, K. Role of mexA-mexB-oprM in antibiotic efflux in Pseudomonas aeruginosa. Antimicrob Agents Chemother 39, 1948-1953, doi:10.1128/aac.39.9.1948 (1995).

$4 \quad$ Li, X. Z., Zhang, L., Srikumar, R. \& Poole, K. Beta-lactamase inhibitors are substrates for the multidrug efflux pumps of Pseudomonas aeruginosa. Antimicrob Agents Chemother 42, 399-403 (1998).

5 Masuda, N. \& Ohya, S. Cross-resistance to meropenem, cephems, and quinolones in Pseudomonas aeruginosa. Antimicrob Agents Chemother 36, 1847-1851, doi:10.1128/aac.36.9.1847 (1992).

6 Poole, K., Krebes, K., McNally, C. \& Neshat, S. Multiple antibiotic resistance in Pseudomonas aeruginosa: evidence for involvement of an efflux operon. J Bacteriol 175, 7363-7372, doi:10.1128/jb.175.22.7363-7372.1993 (1993). 
7 Rella, M. \& Haas, D. Resistance of Pseudomonas aeruginosa PAO to nalidixic acid and low levels of beta-lactam antibiotics: mapping of chromosomal genes. Antimicrob Agents Chemother 22, 242-249, doi:10.1128/aac.22.2.242 (1982).

$8 \quad \mathrm{Li}, \mathrm{X}$. Z., Plesiat, P. \& Nikaido, H. The challenge of efflux-mediated antibiotic resistance in Gram-negative bacteria. Clin Microbiol Rev 28, 337-418, doi:10.1128/CMR.00117-14 (2015).

9 Poole, K. Multidrug efflux pumps and antimicrobial resistance in Pseudomonas aeruginosa and related organisms. J Mol Microbiol Biotechnol 3, 255-264 (2001).

10 Paulsen, I. T., Brown, M. H. \& Skurray, R. A. Proton-dependent multidrug efflux systems. Microbiol Rev 60, 575-608 (1996).

11 Guan, L., Ehrmann, M., Yoneyama, H. \& Nakae, T. Membrane topology of the xenobiotic-exporting subunit, MexB, of the MexA,B-OprM extrusion pump in Pseudomonas aeruginosa. J Biol Chem 274, 10517-10522, doi:10.1074/jbc.274.15.10517 (1999).

12 Tseng, T. T. et al. The RND permease superfamily: an ancient, ubiquitous and diverse family that includes human disease and development proteins. $J$ Mol Microbiol Biotechnol 1, 107-125 (1999).

13 Ma, D., Cook, D. N., Hearst, J. E. \& Nikaido, H. Efflux pumps and drug resistance in gram-negative bacteria. Trends Microbiol 2, 489-493 (1994).

14 Nikaido, H. Prevention of drug access to bacterial targets: permeability barriers and active efflux. Science 264, 382-388, doi:10.1126/science.8153625 (1994).

15 Saier, M. H., Jr., Tam, R., Reizer, A. \& Reizer, J. Two novel families of bacterial membrane proteins concerned with nodulation, cell division and transport. $\mathrm{Mol}$ Microbiol 11, 841-847 (1994).

16 Nakae, T. Role of membrane permeability in determining antibiotic resistance in Pseudomonas aeruginosa. Microbiol Immunol 39, 221-229 (1995).

17 Nakajima, A., Sugimoto, Y., Yoneyama, H. \& Nakae, T. Localization of the outer membrane subunit OprM of resistance-nodulation-cell division family multicomponent efflux pump in Pseudomonas aeruginosa. J Biol Chem 275, 3006430068, doi:10.1074/jbc.M005742200 (2000).

18 Ma, D. et al. Molecular cloning and characterization of acrA and acrE genes of Escherichia coli. J Bacteriol 175, 6299-6313 (1993).

19 Murakami, S., Nakashima, R., Yamashita, E. \& Yamaguchi, A. Crystal structure of bacterial multidrug efflux transporter AcrB. Nature 419, 587-593, doi:10.1038/nature01050 (2002).

20 Murakami, S., Nakashima, R., Yamashita, E., Matsumoto, T. \& Yamaguchi, A. Crystal structures of a multidrug transporter reveal a functionally rotating mechanism. Nature 443, 173-179, doi:10.1038/nature05076 (2006).

21 Du, D. et al. Structure of the AcrAB-TolC multidrug efflux pump. Nature 509, 512 515, doi:10.1038/nature13205 (2014).

22 Seeger, M. A. et al. Structural asymmetry of AcrB trimer suggests a peristaltic pump mechanism. Science 313, 1295-1298, doi:10.1126/science.1131542 (2006).

23 Sennhauser, G., Amstutz, P., Briand, C., Storchenegger, O. \& Grutter, M. G. Drug export pathway of multidrug exporter AcrB revealed by DARPin inhibitors. PLoS Biol 5, e7, doi:10.1371/journal.pbio.0050007 (2007). 
24 Seeger, M. A. et al. Engineered disulfide bonds support the functional rotation mechanism of multidrug efflux pump AcrB. Nat Struct Mol Biol 15, 199-205, doi:10.1038/nsmb.1379 (2008).

25 Takatsuka, Y. \& Nikaido, H. Covalently linked trimer of the AcrB multidrug efflux pump provides support for the functional rotating mechanism. J Bacteriol 191, 17291737, doi:10.1128/JB.01441-08 (2009).

26 Takatsuka, Y. \& Nikaido, H. Site-directed disulfide cross-linking shows that cleft flexibility in the periplasmic domain is needed for the multidrug efflux pump AcrB of Escherichia coli. J Bacteriol 189, 8677-8684, doi:10.1128/JB.01127-07 (2007).

27 Schulz, R., Vargiu, A. V., Collu, F., Kleinekathofer, U. \& Ruggerone, P. Functional rotation of the transporter AcrB: insights into drug extrusion from simulations. PLoS Comput Biol 6, e1000806, doi:10.1371/journal.pcbi.1000806 (2010).

28 Yao, X. Q., Kenzaki, H., Murakami, S. \& Takada, S. Drug export and allosteric coupling in a multidrug transporter revealed by molecular simulations. Nat Commun 1, 117, doi:10.1038/ncomms1116 (2010).

29 Tornroth-Horsefield, S. et al. Crystal structure of AcrB in complex with a single transmembrane subunit reveals another twist. Structure 15, 1663-1673, doi:10.1016/j.str.2007.09.023 (2007).

30 Wang, Z. et al. An allosteric transport mechanism for the AcrAB-TolC multidrug efflux pump. Elife 6, doi:10.7554/eLife.24905 (2017).

31 Vargiu, A. V. \& Nikaido, H. Multidrug binding properties of the AcrB efflux pump characterized by molecular dynamics simulations. Proc Natl Acad Sci U S A 109, 20637-20642, doi:10.1073/pnas.1218348109 (2012).

32 Bohnert, J. A. et al. Site-directed mutagenesis reveals putative substrate binding residues in the Escherichia coli RND efflux pump AcrB. J Bacteriol 190, 8225-8229, doi:10.1128/JB.00912-08 (2008).

33 Bohnert, J. A., Schuster, S., Fahnrich, E., Trittler, R. \& Kern, W. V. Altered spectrum of multidrug resistance associated with a single point mutation in the Escherichia coli RND-type MDR efflux pump YhiV (MdtF). J Antimicrob Chemother 59, 1216-1222, doi:10.1093/jac/dkl426 (2007).

34 Bohnert, J. A., Karamian, B. \& Nikaido, H. Optimized Nile Red efflux assay of AcrABTolC multidrug efflux system shows competition between substrates. Antimicrob Agents Chemother 54, 3770-3775, doi:10.1128/AAC.00620-10 (2010).

35 Wehmeier, C., Schuster, S., Fahnrich, E., Kern, W. V. \& Bohnert, J. A. Site-directed mutagenesis reveals amino acid residues in the Escherichia coli RND efflux pump AcrB that confer macrolide resistance. Antimicrob Agents Chemother 53, 329-330, doi:10.1128/AAC.00921-08 (2009).

36 Vargiu, A. V. et al. Effect of the F610A mutation on substrate extrusion in the AcrB transporter: explanation and rationale by molecular dynamics simulations. J Am Chem Soc 133, 10704-10707, doi:10.1021/ja202666x (2011).

37 Nakashima, R., Sakurai, K., Yamasaki, S., Nishino, K. \& Yamaguchi, A. Structures of the multidrug exporter AcrB reveal a proximal multisite drug-binding pocket. Nature 480, 565-569, doi:10.1038/nature10641 (2011).

38 Eicher, T. et al. Transport of drugs by the multidrug transporter AcrB involves an access and a deep binding pocket that are separated by a switch-loop. Proc Natl Acad Sci U S A 109, 5687-5692, doi:10.1073/pnas.1114944109 (2012). 
39 Yu, E. W., Aires, J. R., McDermott, G. \& Nikaido, H. A periplasmic drug-binding site of the AcrB multidrug efflux pump: a crystallographic and site-directed mutagenesis study. J Bacterio/ 187, 6804-6815, doi:10.1128/JB.187.19.6804-6815.2005 (2005).

40 Husain, F. \& Nikaido, H. Substrate path in the AcrB multidrug efflux pump of Escherichia coli. Mol Microbiol 78, 320-330, doi:10.1111/j.1365-2958.2010.07330.x (2010).

41 Nikaido, H. Structure and mechanism of RND-type multidrug efflux pumps. Adv Enzymol Relat Areas Mol Biol 77, 1-60 (2011).

42 Tsukagoshi, N. \& Aono, R. Entry into and release of solvents by Escherichia coli in an organic-aqueous two-liquid-phase system and substrate specificity of the AcrAB-TolC solvent-extruding pump. J Bacteriol 182, 4803-4810 (2000).

43 White, D. G., Goldman, J. D., Demple, B. \& Levy, S. B. Role of the acrAB locus in organic solvent tolerance mediated by expression of marA, soxS, or robA in Escherichia coli. J Bacteriol 179, 6122-6126 (1997).

44 Eicher, T. et al. Coupling of remote alternating-access transport mechanisms for protons and substrates in the multidrug efflux pump AcrB. Elife 3, doi:10.7554/eLife.03145 (2014).

45 Vargiu, A. V., Ruggerone, P., Opperman, T. J., Nguyen, S. T. \& Nikaido, H. Molecular mechanism of MBX2319 inhibition of Escherichia coli AcrB multidrug efflux pump and comparison with other inhibitors. Antimicrob Agents Chemother 58, 6224-6234, doi:10.1128/AAC.03283-14 (2014).

46 Takatsuka, Y. \& Nikaido, H. Threonine-978 in the transmembrane segment of the multidrug efflux pump AcrB of Escherichia coli is crucial for drug transport as a probable component of the proton relay network. J Bacteriol 188, 7284-7289, doi:10.1128/JB.00683-06 (2006).

47 Case, D. A. et al. AMBER 12. University of California, San Francisco (2012).

48 Nissink, J. W. et al. A new test set for validating predictions of protein-ligand interaction. Proteins 49, 457-471, doi:10.1002/prot.10232 (2002).

49 Nakashima, R. et al. Structural basis for the inhibition of bacterial multidrug exporters. Nature 500, 102-106, doi:10.1038/nature12300 (2013).

50 Case, D. A. et al. The Amber biomolecular simulation programs. J Comput Chem 26, 1668-1688, doi:10.1002/jcc.20290 (2005).

51 Jo, S., Kim, T., Iyer, V. G. \& Im, W. CHARMM-GUI: a web-based graphical user interface for CHARMM. J Comput Chem 29, 1859-1865, doi:10.1002/jcc.20945 (2008).

52 Dickson, C. J. et al. Lipid14: The Amber Lipid Force Field. J Chem Theory Comput 10, 865-879, doi:10.1021/ct4010307 (2014).

53 Hopkins, C. W., Le Grand, S., Walker, R. C. \& Roitberg, A. E. Long-Time-Step Molecular Dynamics through Hydrogen Mass Repartitioning. J Chem Theory Comput 11, 1864-1874, doi:10.1021/ct5010406 (2015).

54 Maier, J. A. et al. ff14SB: Improving the Accuracy of Protein Side Chain and Backbone Parameters from ff99SB. J Chem Theory Comput 11, 3696-3713, doi:10.1021/acs.jctc.5b00255 (2015).

55 Daidone, I. \& Amadei, A. Essential dynamics: foundation and applications. WIRES Comput Mol Sci 2, 762-770, doi:10.1002/wcms.1099 (2012).

56 Garcia, A. E. Large-amplitude nonlinear motions in proteins. Phys Rev Lett 68, 26962699, doi:10.1103/PhysRevLett.68.2696 (1992). 
650

657

57 Thomas, J. R., Gedeon, P. C., Grant, B. J. \& Madura, J. D. LeuT conformational sampling utilizing accelerated molecular dynamics and principal component analysis. Biophys J 103, L1-3, doi:10.1016/j.bpj.2012.05.002 (2012).

58 Gedeon, P. C., Thomas, J. R. \& Madura, J. D. Accelerated molecular dynamics and protein conformational change: a theoretical and practical guide using a membrane embedded model neurotransmitter transporter. Methods Mol Biol 1215, 253-287, doi:10.1007/978-1-4939-1465-4_12 (2015).

\section{Acknowledgements}

We thank Mr Daniel Mikolajczyk, IT Systems Administrator of King's College London, for installing and supporting FWB-Lab-Tesla HPC system on the Rahman research group's supercomputer (Tesla Biohealth Lab). This work was funded by Grants from National Biofilm Innovation Centre (POC2-091) and BBSRC (BB/T007737/1). We are also thankful to Dr Mark Laws for proofreading an earlier version of the manuscript.

\section{Author contribution}

SJ, KMR and JMS conceived the study. SJ designed and performed the study and wrote the manuscript. SJ contributed to data analysis. SJ wrote the manuscript, KMR and JMS edited the manuscript.

\section{Conflicts of Interests}

The authors have no conflict of interest to declare.

\section{Data Availability Statement}

All data generated or analysed during this study are included in this published article (and its Supplementary Information files). 
660

661

662

\begin{tabular}{|c|c|c|c|c|c|c|c|c|c|c|}
\hline \multicolumn{2}{|c|}{ Distance (A) with highest density during MD } & \multicolumn{3}{|l|}{ MexB } & \multicolumn{3}{|c|}{ MexB-PABN } & \multicolumn{3}{|c|}{ MexB-TET } \\
\hline TM helix & Residues & Access & Binding & Release & Access & Binding & Release & Access & Binding & Release \\
\hline TM2-TM10 & Glu346_Asp923 & 28.99 & 28.73 & 25.99 & 26.53 & 26.39 & 26.82 & 26.54 & 28.74 & 27.35 \\
\hline TM11-TM2 & Thr976_His338 & 27.92 & 26.14 & 29.57 & 28.17 & 25.51 & 29.54 & 27.92 & 26.65 & 29.69 \\
\hline TM10-TM11 & Lys939_Thr976 & 12.38 & 13.09 & 11.83 & 13.59 & 12.97 & 12.00 & 12.96 & 10.67 & 11.42 \\
\hline TM4-TM10 & Asp408_Asp923 & 28.59 & 28.56 & 26.51 & 29.30 & 27.89 & 26.48 & 29.36 & 28.08 & 26.76 \\
\hline TM10-TM11 & Asp923_Thr976 & 28.1 & 58.63 & 57.68 & 28.39 & 58.71 & 56.51 & 28.02 & 58.52 & 56.76 \\
\hline TM4-TM2 & Asp408_His338 & 28.4 & 25.58 & 27.21 & 27.36 & 25.26 & 27.03 & 27.42 & 25.59 & 27.11 \\
\hline Postulate gate & Gln125_Tyr757 & 9.20 & 9.10 & 18.45 & 9.64 & 7.90 & 15.97 & 8.44 & 7.67 & 16.24 \\
\hline Cleft & Phe664_Arg716 & 11.32 & 13.95 & 8.86 & 11.00 & 12.41 & 8.64 & 11.95 & 10.07 & 9.53 \\
\hline
\end{tabular}

663

664

665

666

667

668

669

670

671

672

673

674

675

676

677

678

679

680 

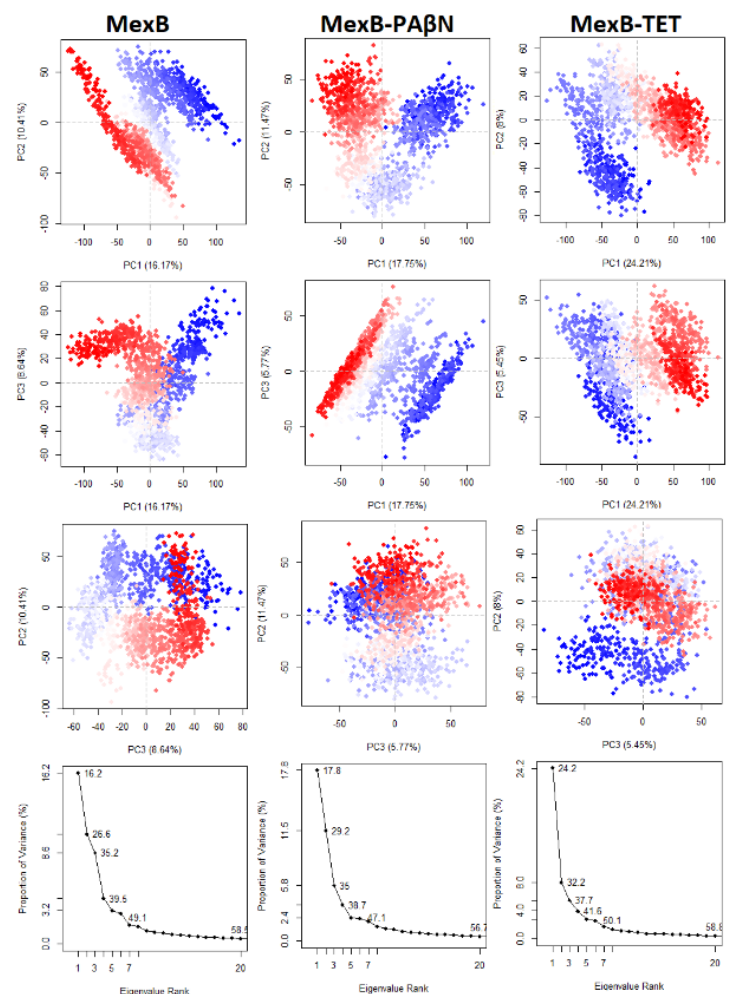

682

Figure 1. Rows 1-3) PCA results for MexB in free form and in complex with PA $\beta N$ and TET trajectories with instantaneous conformations (trajectory frames) coloured from blue to red in order of time. Row 4) The rank ordering of the eigenvalues of the covariance matrix. Eigenvalue spectrum; Results obtained from diagonalization of the atomic displacement correlation matrix of $\mathrm{C} \alpha$ atom coordinates from the first snapshot structures. Inset shows histograms for the projection of the distribution of structures onto the first three principal components. 


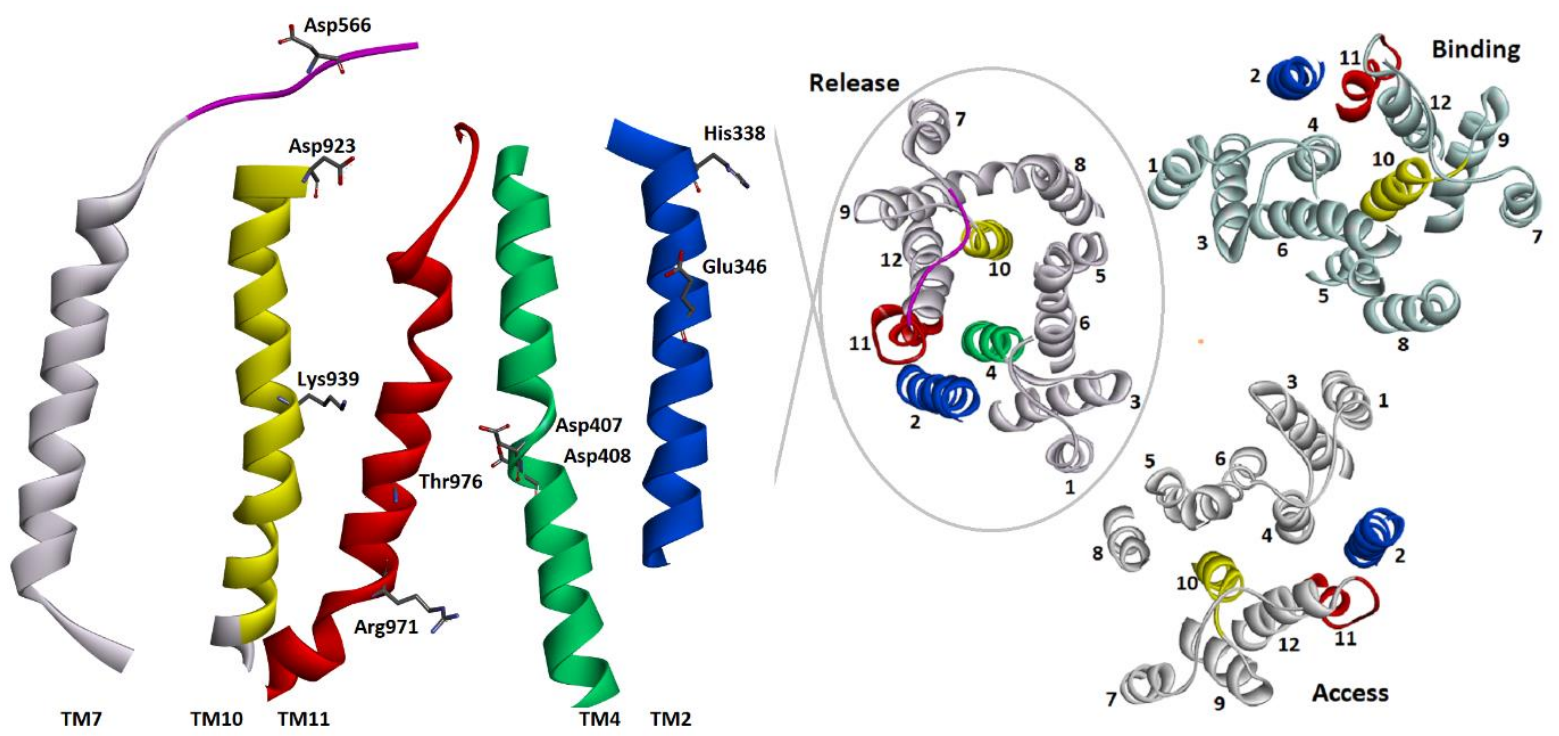

Figure 2. Periplasmic top view of a ribbon representation of the protomers is represented in right panel. The label numbers indicate the transmembrane helix numbers (TMx). The individually colored helices include the protonated residues in each protomer. A side view of the transmembrane domain with protonating residues marked is provided in the left panel as well.

707

708

709 


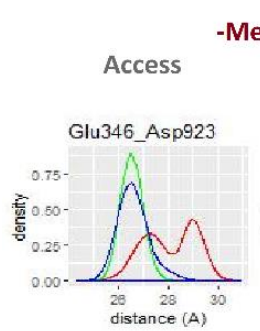

MexB -MexB-PAßN -MexB-TET
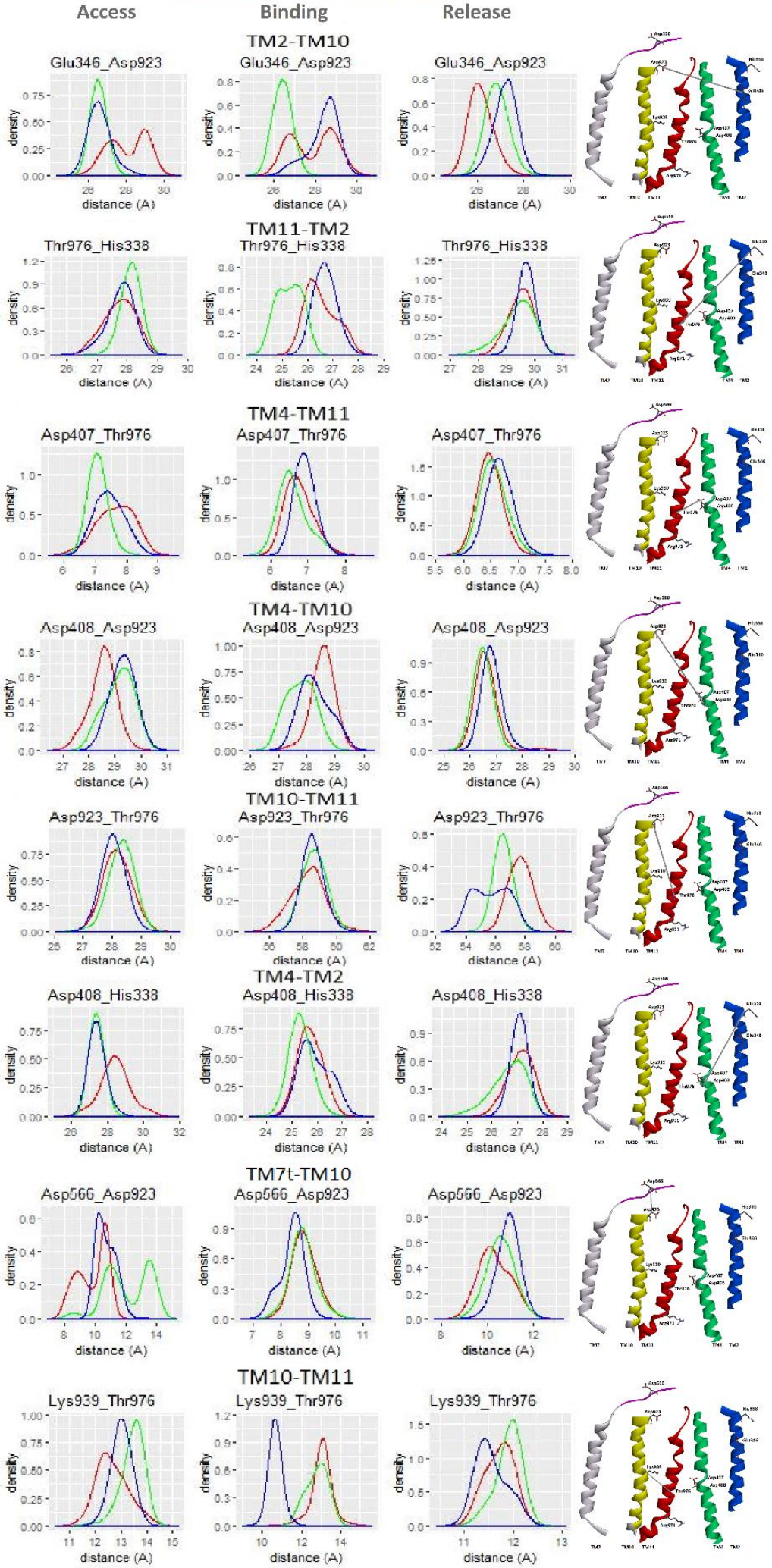

Figure 3. Ligand-dependent conformational changes of MexB in the transmembrane regions (TM2, 4, 10 and 11). Distance distributions showing density versus distance between identical positions in the labelled structure. Distance distributions for each pair were obtained in the ligand-free (red), PABNbound (green) and TET-bound (blue) systems. For clarity, TM helices are shown in expanded form in the right-hand panel 

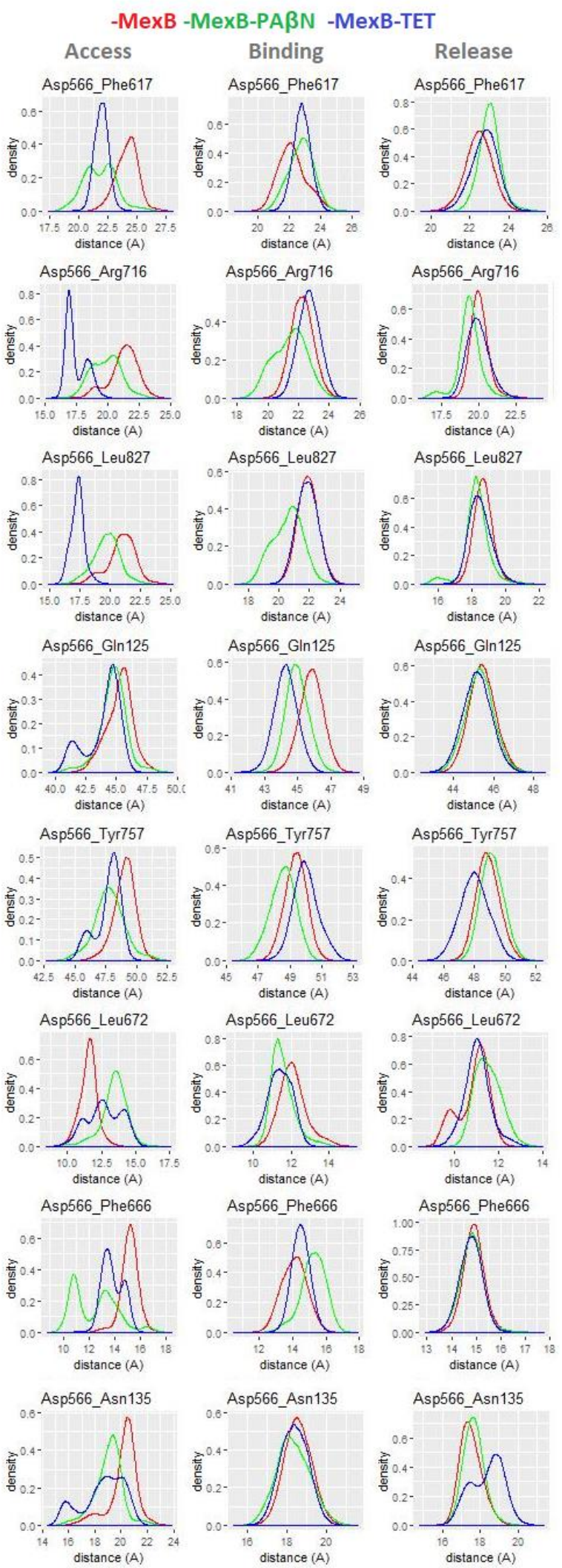

730 Figure 4. Ligand-dependent conformational changes in the distance between Asp566 in the transmembrane regions to the key residues of multi-binding site of MexB. Distance distributions show the density versus distance between identical amino acid positions and Asp566. Distance distributions were obtained in the ligand free (red), PABN-bound (green) and TET-bound (blue) systems. 
-MexB-PAßN -MexB-TET
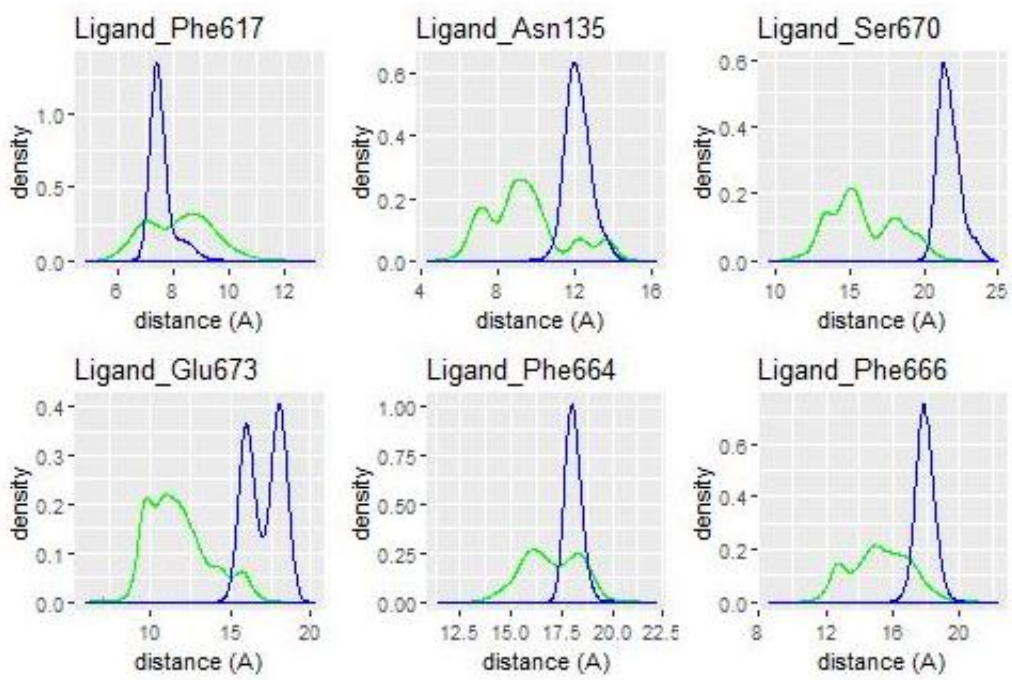

Figure 5. Density of distance distribution in terms of the distance between the ligands and diverse key residues of passageway of multi-binding site in binding protomer of MexB-PAßN (green) and MexBTET (blue) complexes as calculated from MD trajectories. Distance distributions show density versus distance between identical positions from the bound ligands.

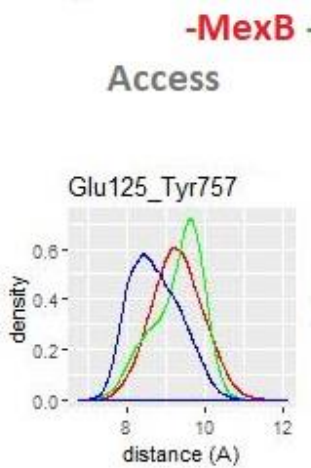

\section{-MexB-PAßN -MexB-TET}

Binding

Release

\section{Postulate gate}
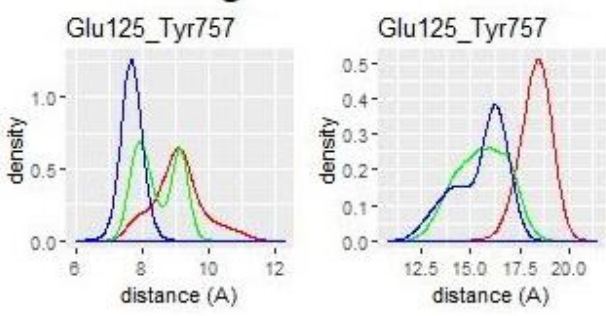

Cleft
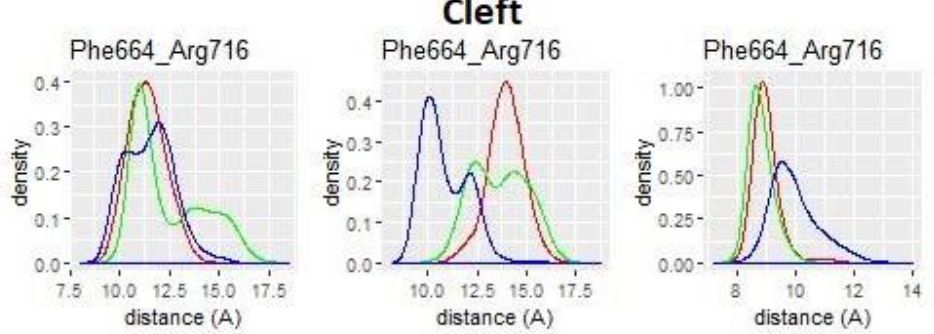

Figure 6. Density of distance distribution between the postulated gate residues and cleft residues in diverse protomers of MexB (red), MexB-PABN (green) and MexB-TET (blue) complexes as calculated from MD trajectories. Distance distributions depict the density of a distance versus distance between identical position of Gln125 and Tyr757 and Phe664 and Arg716 as representative residues of postulated gate and cleft in diverse monomers of the different systems. 